\title{
Sub-band Equalization Filter Design for Improving Dynamic Range Performance of Modulated Wideband Converter
}

\author{
Yasar Kemal Alp*, Gokhan Gok *† Ali Bugra Korucu* ${ }^{* \ddagger}$ \\ ${ }^{*}$ Radar Electronic Warfare and Intelligence Systems Division, ASELSAN A.S., Ankara, Turkey \\ $\dagger$ Electrical and Electronics Engineering Department, Bilkent University, Ankara, Turkey \\ ‡Electrical and Electronics Engineering Department, TOBB ETU, Ankara, Turkey \\ \{ykalp,gokhangok,abkorucu\}@ aselsan.com.tr
}

\begin{abstract}
In this work, we propose an iterative method to improve the dynamic range performance of the Modulated Wideband Converter (MWC), which is multi-channel sampling system for digitizing wideband sparse signals below the Nyquist limit without loss of information by using compressive sensing techniques. Our method jointly designs FIR filters for each subband to equalize the frequency response characteristics of the all sub-bands of the MWC. Obtained results from the extensive computer simulations of the MWC system show that the proposed method improves the dynamic range performance of the MWC system significantly.
\end{abstract}

\section{INTRODUCTION}

Modulated Wideband Converter (MWC) is a compressive sensing based, multichannel sub-Nyquist sampling system for digitizing frequency sparse wideband signals without loss of information [1], [2]. The MWC system, whose block diagram is shown in Fig.1, assumes that the signal of interest is a multi-band signal [5], i.e., the signal is composed of many sub-bands and only a small fraction of the sub-bands are active for a limited observation time. First, the incoming signal is divided into a number of identical analog channels by means of a power divider. In each analog channel, the signal is mixed with a different periodic waveform, which has many harmonics with different magnitude and phase values throughout the total bandwidth of the signal. This mixing operation generates different linear combinations of the subbands at the baseband as well as the harmonic freuquencies of the mixing waveform. Hence, in each channel a narrowband compressed spectrum is generated. By using the narrowband sub-band mixtures coming from different channels, active subbands in the incoming signal are detected. Moreover, baseband representations of the active sub-bands are reconstructed.

For successful active sub-band detection, the number of channels should be chosen according to the sparsity level of the multiband signal of interest. In [1], authors showed that the number of channels should be at least 2 times the number of active sub-bands. For instance, if there are three real narrowband transmissions, then at least 6 bands are active (counting active bands for both positive and negative freuqencies). Moreover, if the these transmissions are distributed over two neighbouring sub-bands, 12 sub-bands would be active, which would require 24 sampling channels for successful recovery. In real hardware implementations, a receiver with 24 analog channels is infeasible because of size/weight/power/cost considerations. To decrease the number of required analog channels, in [1] authors propose to expand the number of channels by a factor of $q$, by applying digital filtering to $q$ sub-bands of each analog channel. Since the analog filter response in each analog channel is never ideal, the frequency response of the $q$ synthesized sub-band channels by digital filtering would not be identical. The frequency response difference between these channels significantly degrades the dynamic range performance of the system.

In this work, we propose an iterative method for equalizing the frequency response characteristics of sub-band channels of the MWC for improved dynamic range performance that makes MWC usable in practical applications. Our method is based on FIR filter design, where in each iteration, we design separate FIR filters for each sub-band channel and update the total channel response. After a few iterations, the method converges and the resulting frequency response of the sub-band channels are equalized. Simulation results show that proposed sub-band equalization method significantly improves the dynamic range performance of the MWC.

The organization of the paper is as follows. In SectionII, the MWC will be reviewed. In Section-III, proposed subband equalization method is detailed. Simulation results are provided in Section-IV. Concluding remarks are given in Section-V. Throughout the paper, bold small and bold capital characters will denote vectors and matrices, respectively.

\section{MWC: Modulated WideBAnd CONVERTER}

Let $\mathcal{F}=\left[-F_{N y q} / 2, F_{N y q} / 2\right]$ be the operating frequency range of the MWC system whose block diagram is given in Fig.1. Assume that this range has been divided into $2 L+1$ subbands of bandwidth $B$. Each sub-band has the following frequency support $\mathcal{F}_{l}=[l B-B / 2, l B+B / 2], l=-L, . ., L$, where the first and the last sub-bands satisfy the following conditions $-L B-B / 2 \geq-F_{N y q} / 2$ and $L B+B / 2 \leq F_{N y q} / 2$, respectively. 


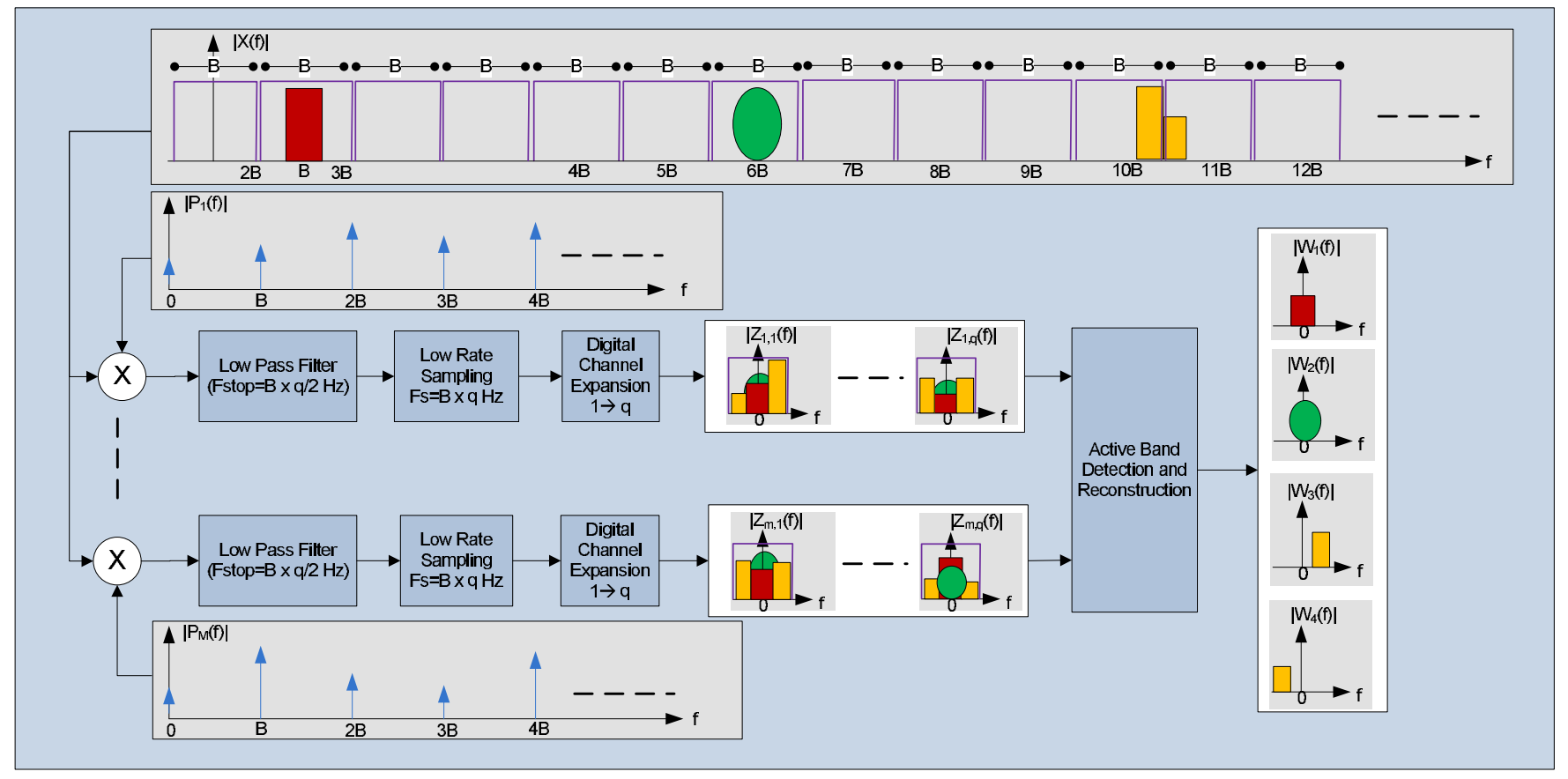

Fig. 1. Modulated Wideband Converter

Consider the following multi-band measurement model, which has $P$ active bands:

$$
x(t)=\sum_{p=1}^{P} a_{p}(t) e^{j 2 \pi B l_{p} t} .
$$

Here $l_{p} \in[-L, L]$ and $a_{p}(t)$ is the complex bandlimited signal satisfying $A_{p}(f)=0, \forall|f| \geq B / 2$, where $A_{p}(f)$ is the Fourier transform of $a_{p}(t)$ defined as $A_{p}(f)=\int_{-\infty}^{\infty} a_{p}(t) e^{-j 2 \pi f t} d t$. Once $x(t)$ is received by the MWC, it is divided and fed into $M$ identical analog channels, where in each channel $x(t)$ is multiplied by the real and periodic waveforms $p_{m}(t), m=1, \ldots, M$. These waveforms are different from each other but they have the same period $T_{p}=1 / B$. Hence, each waveform have the following Fourier series expansion:

$$
p_{m}(t)=\sum_{k=-\infty}^{\infty} c_{m, k} e^{j 2 \pi k B t}
$$

where $c_{m, k}$ is the $k^{\text {th }}$ Fourier series coefficient of the $m^{\text {th }}$ waveform, which can be computed as $c_{m, k}=B \int_{t_{0}}^{t 0+1 / B} p_{m}(t) e^{-j 2 \pi k B t} d t$ for any time instant $t_{0}$. Note that $c_{m, k}=c_{m, k}^{*}$, since $p_{m}(t)$ is real. The representative spectra of $p_{m}(t)$ are shown as $P_{m}(f)$ in Fig.1. In each analog channel, before the analog low-pass filter the following signal appears:

$$
y_{m}(t)=x(t) p_{m}(t)=\sum_{k=-\infty}^{\infty} \sum_{p=1}^{P} c_{m,-l_{p}+k} a_{p}(t) e^{j 2 \pi k B t} .
$$

If the stop band frequency of the identical analog low-pass filters in each channel is chosen as $q \times B / 2$, where $q$ being the channel expansion factor [1], the resulting signals $v_{m}(t)$ at the end of each filter can be written as:

$$
v_{m}(t)=\sum_{k=-\hat{q}}^{\hat{q}} \sum_{p=1}^{P} c_{m,-l_{p}+k}\left(a_{p}(t) e^{j 2 \pi k B t}\right) * h_{A}(t),
$$

where $\hat{q}=(q-1) / 2$ and $h_{A}(t)$ is the impulse response of the analog filter. In (4), we assume that $H_{A}(f)=0 \forall|f| \geq B q / 2$, where $H_{A}(f)$ is the Fourier transform of $h_{A}(t)$. In the Fourier domain, (4) has the following equivalent representation

$$
V_{m}(f)=\sum_{k=-\hat{q}}^{\hat{q}} \sum_{p=1}^{P} c_{m,-l_{p}+k} A_{p}(f+k B) H_{A}(f),
$$

where $V_{m}(f)$ is the Fourier transforms of $v_{m}(t)$ respectively. Note that different linear combinations of $a_{p}(t), p=1, \ldots, P$ signals appear at centre frequencies $k B, k=-\hat{q}, . ., \hat{q}$ in $v_{m}(t)$. To expand the number of channels by a factor of $q$, each channel is sampled at a rate $F_{s} \geq q B$ and multiplied by $e^{j 2 \pi q^{\prime} B n / F_{s}}, q^{\prime}=-\hat{q}, . ., \hat{q}$ and a digital low-pass filter with cut-off frequency $B / 2$ is applied. Hence, from each analog channel, the following $q$ digital sub-band channel signals are generated:

$$
v_{m, q^{\prime}}[n]=\left(v_{m}\left(n T_{s}\right) e^{j 2 \pi q^{\prime} B n T_{s}}\right) * h_{D}[n], q^{\prime}=-\hat{q}, . ., \hat{q},
$$

where $T_{s}=1 / F_{s}$ is the sampling period of the ADC and $h_{D}[n]$ denote the digital filter coefficients. (6) can be equivalently written in the spectral domain as:

$$
\begin{array}{r}
V_{m, q^{\prime}}(f)=\sum_{p=1}^{P} c_{m,-l_{n}+q^{\prime}} A_{p}(f) H_{A}\left(f-q^{\prime} B\right) H_{D}(f), \\
|f| \leq F_{s} / 2, q^{\prime}=-\hat{q}, \ldots, \hat{q} .
\end{array}
$$


Here $V_{m, q^{\prime}}(f)$ is the Fourier transform of the digital signal coming from the $q^{\text {th }}$ digital sub-band channel of the $m^{\text {th }}$ analog channel and $H_{D}(f)$ is the frequency response of the digital filter. Assuming that both analog and digital filters are ideal, i.e.

$$
\begin{aligned}
& H_{A}(f)= \begin{cases}1 & \text { if }|f| \leq q B / 2, \\
0 & \text { otherwise }\end{cases} \\
& H_{D}(f)= \begin{cases}1 & \text { if }|f| \leq B / 2 \\
0 & \text { otherwise }\end{cases}
\end{aligned}
$$

all the $M \times q$ channels have identical frequency response hence (7) can be written as the following linear system of equations:

$$
\mathbf{v}(f)=\mathbf{C b}(f)
$$

where

$$
\begin{aligned}
\mathbf{C}_{M q \times 2 L+1}= & {\left[\begin{array}{cccc}
c_{1,-L-\hat{q}} & c_{1,-L+1-\hat{q}} & . . & c_{1, L-\hat{q}} \\
c_{1,-L-\hat{q}+1} & c_{1,-L+1-\hat{q}+1} & . . & c_{1, L-\hat{q}+1} \\
\cdot & \cdot & . . & \cdot \\
c_{1,-L+\hat{q}} & c_{1,-L+1+\hat{q}} & . . & c_{1, L+\hat{q}} \\
c_{2,-L-\hat{q}} & c_{2,-L+1-\hat{q}} & . . & c_{2, L-\hat{q}} \\
\cdot & \cdot & . \cdot & \cdot \\
\cdot & \cdot & . \cdot & \cdot \\
c_{M,-L+\hat{q}} & c_{M,-L+1+\hat{q}} & . \cdot & c_{M, L+\hat{q}}
\end{array}\right], } \\
\mathbf{v}_{M q \times 1}(f)= & {\left[\begin{array}{c}
V_{1,-\hat{q}}(f) \\
V_{1,-\hat{q}+1}(f) \\
\cdot \\
V_{1, \hat{q}}(f) \\
V_{2,-\hat{q}}(f) \\
\cdot \\
\cdot \\
V_{M}
\end{array}\right], \mathbf{b}_{2 L+1 \times 1}(f)=\left[\begin{array}{c}
\cdot \\
\cdot \\
b_{1}(f) \\
b_{2 L+1}(f)
\end{array}\right], }
\end{aligned}
$$

where $\mathbf{b}(f)$ is $P$-sparse, with the following non-zero entries $\mathbf{b}_{l_{p}+L+1}(f)=A_{p}(f), p=1, . ., P$. For the discrete set of frequency values $f_{n}, n=0,1, . ., N-1$, where $f_{n}=F_{s} n / N$, a multiple measurement formulation of (10) can be constructed as:

$$
\mathbf{V}=\mathbf{C B}
$$

where $\mathbf{V}=\left[\mathbf{v}\left(f_{0}\right), . ., \mathbf{v}\left(f_{N-1}\right)\right]$ and $\mathbf{B}=\left[\mathbf{b}\left(f_{0}\right), . ., \mathbf{b}\left(f_{N-1}\right)\right]$. Given the multiple measurement vector $\mathbf{V}$ and the system matrix C, the sparsest (block sparsity) B satisfying (12) is to be found. There are many fast greedy algorithms for solving (12) [3], [4]. Note that, by multiplying both sides of (12) with the inverse DFT matrix from the right, would enable to work with time domain samples rather than the spectral slices.

\section{Digitial Filter Design FOR Sub-BAnd EQUALIZATION OF MWC}

In real implementations, the analog low-pass filter is never ideal. Hence, the total filter responses in the sub-bands

$$
H_{q^{\prime}}(f)=H_{A}\left(f-q^{\prime} B\right) H_{D}(f), q^{\prime}=-\hat{q}, . ., \hat{q}
$$

are not identical. Hence the linear system in (10) can not be written directly. To equalize the sub-band channel frequency responses, we propose an iterative method to design separate FIR filters for each sub-band. Consider the following minimization problem at the $i^{t h}$ iteration of the proposed method:

$$
\begin{aligned}
& \underset{\substack{\mathbf{w}_{q^{\prime}} \\
q^{\prime}=-\hat{q}, . ., \hat{q}}}{\arg \min } \nu\left(\sum_{\substack{\mathbf{w}_{q^{\prime} \in \mathcal{C}^{K}}, q^{\prime}=-\hat{q}, . ., \hat{q}}} \int_{\substack{k \neq l \\
k, l=-\hat{q}, . ., \hat{q}^{-}}}^{F_{s} / 2} \mid H_{k}(f) H_{E}\left(f ; \mathbf{w}_{\mathbf{k}}\right)\right. \\
& \left.-\left.H_{l}(f) H_{E}\left(f ; \mathbf{w}_{l}\right)\right|^{2} d f\right) \\
& +(1-\nu)\left(\sum_{q^{\prime}=-\hat{q}_{-}}^{\hat{q}} \int_{F_{s} / 2}^{F_{s} / 2}\left|H_{q^{\prime}}(f) H_{E}\left(f ; \mathbf{w}_{q^{\prime}}\right)-M^{i}(f)\right|^{2} d f\right)
\end{aligned}
$$

where $\mathbf{w}_{q^{\prime}} \in \mathcal{C}^{K}$ are the complex filter coefficients to be designed for the $q^{\text {th }}$ sub-band channel, $M^{i}(f)$ is the filter mask that is used at the $i^{\text {th }}$ iteration and $0<\nu<1$ is the tradeoff parameter. $H_{E}\left(f ; \mathbf{w}_{q^{\prime}}\right)$ is the frequency response of $\mathbf{w}_{q^{\prime}}$ given by

$$
H_{E}\left(f ; \mathbf{w}_{q^{\prime}}\right)=\mathbf{r}(f)^{H} \mathbf{w}_{q^{\prime}}
$$

where $\mathbf{r}(f)=\left[1, e^{-j 2 \pi f / F_{s}}, e^{-j 2 \pi 2 f / F_{s}}, . ., e^{-j 2 \pi(K-1) f / F_{s}}\right]^{H}$. In the first part of cost function of the optimization problem in (14), the energy of the difference between the frequency responses of all sub-bands is minimized. In the second part, we minimize the energy of the difference between each sub-band and the mask. Note that, without the second part, minimization of the first part would yield the trivial solution $\mathbf{w}_{q^{\prime}}=\mathbf{0}, \forall q^{\prime}=-\hat{q}, . ., \hat{q}$.

To simplify the notation, we stack $\mathbf{w}_{q^{\prime}}, q^{\prime}=-\hat{q}, . ., \hat{q}$ on top of each other and define a single optimization variable $\mathbf{w}=\left[\mathbf{w}_{-\hat{q}}^{T}, . ., \mathbf{w}_{\hat{q}}^{T}\right]^{T} \in \mathcal{C}^{K q}$. Also we define diagonal suband selection matrices $\mathbf{S}_{q^{\prime}} \in \mathcal{C}^{K q \times K q}, q^{\prime}=-\hat{q}, . ., \hat{q}$, whose $\left(q^{\prime}+\hat{q}\right) K+1, . .,\left(q^{\prime}+\hat{q}\right) K+K$ diagonal elements are 1 , remaining ones are 0 . Then (14) takes the following form:

$$
\begin{aligned}
& \tilde{\mathbf{w}}^{i}=\underset{\mathbf{w} \in \mathcal{C}^{K q}}{\arg \min } \nu\left(\sum_{\substack{k \neq l \\
k, l=-\hat{q}, . ., \hat{q}^{-F_{s} / 2}}} \int^{F_{s} / 2} \mid\left[H_{k}(f) \mathbf{r}(f)^{H} \mathbf{S}_{k}\right.\right. \\
& \left.\left.-H_{l}(f) \mathbf{r}(f)^{H} \mathbf{S}_{l}\right]\left.\mathbf{w}\right|^{2} d f\right) \\
& +(1-\nu)\left(\sum_{q^{\prime}=-\hat{q}_{-}} \int_{F_{s} / 2}^{F_{s} / 2}\left|H_{q^{\prime}}(f) \mathbf{r}(f)^{H} \mathbf{S}_{q^{\prime}} \mathbf{w}-M^{i}(f)\right|^{2} d f\right) .
\end{aligned}
$$

We further define $\mathbf{x}_{k, l}(f)^{H}=H_{k}(f) \mathbf{r}(f)^{H} \mathbf{S}_{k}-H_{l}(f) \mathbf{r}(f)^{H} \mathbf{S}_{l}$ 
and $\mathbf{x}_{q^{\prime}}(f)=H_{q^{\prime}}(f) \mathbf{r}(f)^{H} \mathbf{S}_{q^{\prime}}$ and rewrite (16) as:

$$
\begin{aligned}
& \tilde{\mathbf{w}}^{i}=\underset{\mathbf{w} \in \mathcal{C}^{K q}}{\arg \min } \mathbf{w}^{H}\left[\nu \sum_{k \neq l} \int_{-F_{s} / 2}^{F_{s} / 2} \mathbf{x}_{k, l}(f) \mathbf{x}_{k, l}(f)^{H} d f\right] \mathbf{w} \\
& +\mathbf{w}^{H}\left[(1-\nu) \sum_{q^{\prime}=-\hat{q}_{-}}^{\hat{q}} \int_{F_{s} / 2}^{F_{s} / 2} \mathbf{x}_{q^{\prime}}(f) \mathbf{x}_{q^{\prime}}(f)^{H} d f\right] \mathbf{w} \\
& -\left[(1-\nu) \sum_{q^{\prime}=-\hat{q}_{-} F_{s} / 2}^{F_{s} / 2} M^{i}(f)^{*} \mathbf{x}_{q^{\prime}}(f)^{H} d f\right] \mathbf{w} \\
& -\mathbf{w}^{H}\left[\begin{array}{c}
\left.(1-\nu) \sum_{q^{\prime}=-\hat{q}_{-}}^{F_{F_{s}} / 2} \mathbf{x}_{q^{\prime}}(f) M^{i}(f) d f\right] \\
\int_{F_{s} / 2}\left|M^{i}(f)\right|^{2} d f .
\end{array}\right. \\
& +q(1-\nu)
\end{aligned}
$$

The cost function in (17) can be written in the following compact form:

$$
\tilde{\mathbf{w}}^{i}=\underset{\mathbf{w} \in \mathcal{C}^{K q}}{\arg \min } \mathbf{w}^{H} \mathbf{X} \mathbf{w}-\mathbf{y}^{i^{H}} \mathbf{w}-\mathbf{w}^{H} \mathbf{y}+c^{i},
$$

where we collapse the sum of the terms in the first and second squared brackets in matrix $\mathbf{X}$, define $\mathbf{y}^{i}$ vector as the term in the fourth squared bracket and assign the value of the last term in (17) to the scaler $c^{i}$. The cost function of the optimization problem in (18) is convex since $\mathbf{X}$ is positive-definite. Hence the optimal $\tilde{\mathbf{w}}^{i}$ which minimizes (18) is given by:

$$
\tilde{\mathbf{w}}^{i}=\left(\mathbf{X X}^{H}\right)^{-1} \mathbf{X} \mathbf{y}^{i} .
$$

Once $\tilde{\mathbf{w}}^{i}$ is found, the optimal filter coefficients for each subband is computed as $\tilde{\mathbf{w}}_{q^{\prime}}^{i}=\mathbf{S}_{q^{\prime}} \tilde{\mathbf{w}}^{i}, q^{\prime}=-\hat{q}, . ., \hat{q}$.

Since the proposed method is iterative, in the initialization step, we form the mask as the mean of the total frequency responses the of all sub-bands:

$$
M^{0}(f)=\sum_{q^{\prime}=-\hat{q}}^{\hat{q}} H_{q^{\prime}}(f) / q .
$$

During the iterations, when the optimal sub-band channel equalizing filter coefficients are found, we update the mask as

$$
M^{i+1}(f)=\sum_{q^{\prime}=-\hat{q}}^{\hat{q}} H_{q^{\prime}}(f) H_{E}\left(f ; \tilde{\mathbf{w}}_{q^{\prime}}^{i}\right) / q .
$$

After a few iterations, estimated filter coefficients $\tilde{\mathbf{w}}_{q^{\prime}}, q^{\prime}=-\hat{q}, . ., \hat{q}$ converge to fixed values and algorithm is terminated.

\section{Simulation Results}

To monitor the proposed sub-band equalization method on the dynamic range performance of the MWC, we simulated the MWC with the simulation parameters provided in Table-I. We
TABLE I

Simulation PaRAMETERS OF THE MOdUlated WidEBAND CONVERTER.

\begin{tabular}{|c|c|c|}
\hline & Value & Notes \\
\hline$F_{N y q}$ & $3000 \mathrm{MHz}$ & Nyquist frequency of the system \\
\hline$M$ & 4 & \# of analog channels \\
\hline$q$ & 7 & Channel expansion factor \\
\hline$B$ & $30 \mathrm{MHz}$ & Bandwidth of each sub-band \\
\hline$F_{s}$ & $250 \mathrm{MHz}$ & Sampling frequency at each analog channel \\
\hline$L$ & 50 & \# sub-bands of bandwidth $30 \mathrm{MHz}$ \\
\hline
\end{tabular}
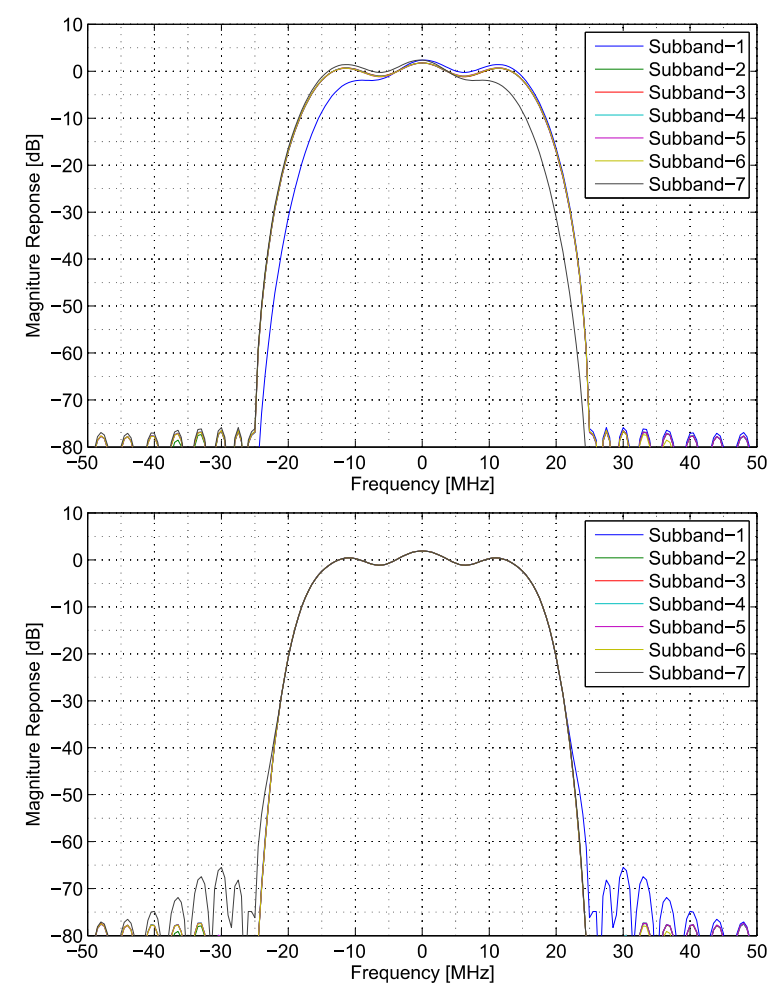

Fig. 2. Total frequency response of the sub-bands before (top) and after (bottom) the proposed sub-band equalization.

used a lengthy (order 1200) equiripple FIR filter to simulate the analog filter $\left(H_{A}(f)\right)$. The filter has $80 \mathrm{~dB}$ attenuation at $125 \mathrm{MHz}$ and its $3 \mathrm{~dB}$ cut-off frequency is $105 \mathrm{MHz}$. To expand the number of channels by a factor 7 , we used a digital filter $\left(H_{D}(f)\right)$ of order 60 , which has $3 \mathrm{~dB}$ cut-off frequency of $15 \mathrm{MHz}$ and $80 \mathrm{~dB}$ attenuation at $25 \mathrm{MHz}$. For sub-band channel equalization, we have designed 7 complex FIR filters, each of which is of order 14 by using the proposed method. The total frequency of the 7 sub-band channels before subband equalization are provided on top of Fig.2. Magnitude response of each sub-band differs from the others. On the bottom of the same figure, the total frequency response of the sub-bands after proposed sub-band equalization is provided. As observed, frequency responses of the all sub-band channels are equalized. Real and imaginary parts of the designed subband equalization filter coefficients are provided in Fig.3.

For analyzing the effect of the proposed method on the 

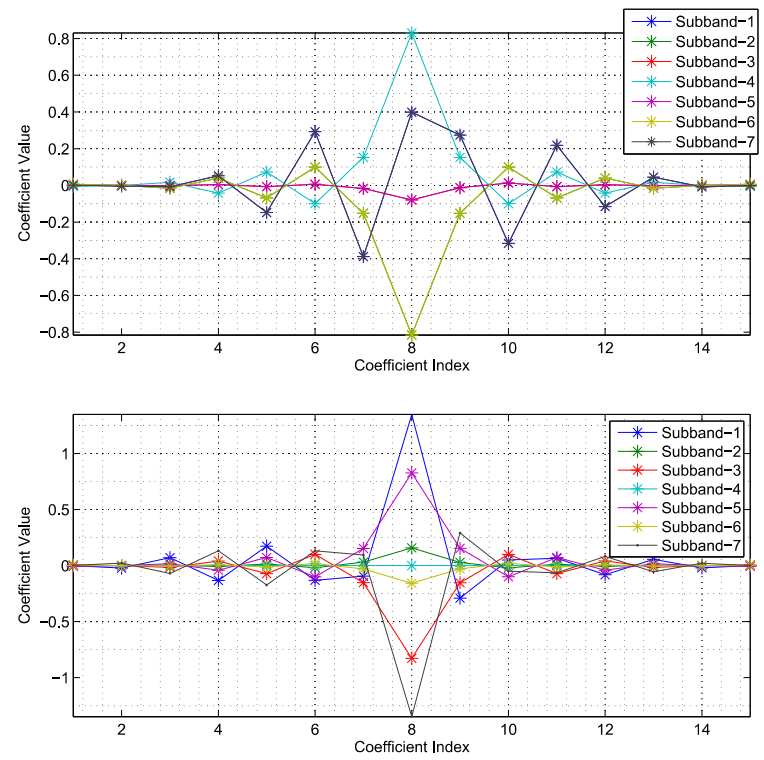

Fig. 3. Real (top) and imaginary (bottom) parts of the designed sub-band equalization filters.

dynamic range of the MWC, the following two tone signal is used:

$$
x(t)=a_{1} \cos \left(2 \pi f_{1} t+\phi_{1}\right)+a_{2} \cos \left(2 \pi f_{2} t+\phi_{2}\right)+n(t) .
$$

Here $a_{i}, f_{i}, \phi_{i}, i=1,2$ are the amplitude levels, center frequencies and phases of the tones, respectively. $n^{r}(t)$ is the white Gaussian noise. We fixed the amplitude level the second sine wave $\left(a_{2}\right)$ such that its power on a 50 ohm resistor is $-70 \mathrm{dBm}$. Note that the noise power after the ADC (in $125 \mathrm{MHz}$ bandwidth) is around $-90 \mathrm{dBm}$. Since incoming signal is divided into 4 channels, observed SNR after the ADC for the second wave is around $14 \mathrm{~dB}$ $\left(20-10 \log _{10}(4)\right)$. We define the dynamic range of $x(t)$ as $D R=20 \log _{10}\left(a_{1} / \sqrt{(2)}\right)-20 \log _{10}\left(a_{2} / \sqrt{(2)}\right)$, assuming that $a_{1}>a_{2}$. For a given dynamic range value, we compute $a_{2}$ accordingly. For different dynamic range values $(D R=10 \mathrm{~dB}, 15 \mathrm{~dB}, . ., 80 \mathrm{~dB})$ and number of samples used for reconstruction $\left(N_{s}=50,100, . ., 400\right)$, we have utilized 1000 Monte-Carlo runs, where in each run $\phi_{1}, \phi_{2}$ are chosen uniformly random from the range $[0,2 \pi] ; f_{1}$ and $f_{2}$ are chosen uniformly random from the range $\left[0, F_{N y q} / 2\right]$. Then (10) is solved for the support recovery. A correct recovery is reported if the recovered sub-band indices includes $\left\lceil f_{1} / B\right\rceil+L$ and $\left\lceil f_{2} / B\right\rceil+L \mathrm{i}$ where $\lceil$.$\rceil is the rounding operation. In Fig.4,$ the probability of correct support recovery as a function of dynamic range and number of samples used for reconstruction without sub-band equalization (top) and with sub-band equalization (bottom) are provided. As observed, when sub-band equalization is not applied, because of the different frequency response characteristics of the sub-bands, dynamic range of the system is limited to $20 \mathrm{~dB}$. However, when the proposed sub-band equalization is applied, the dynamic range of the system achieves $55 \mathrm{~dB}$.
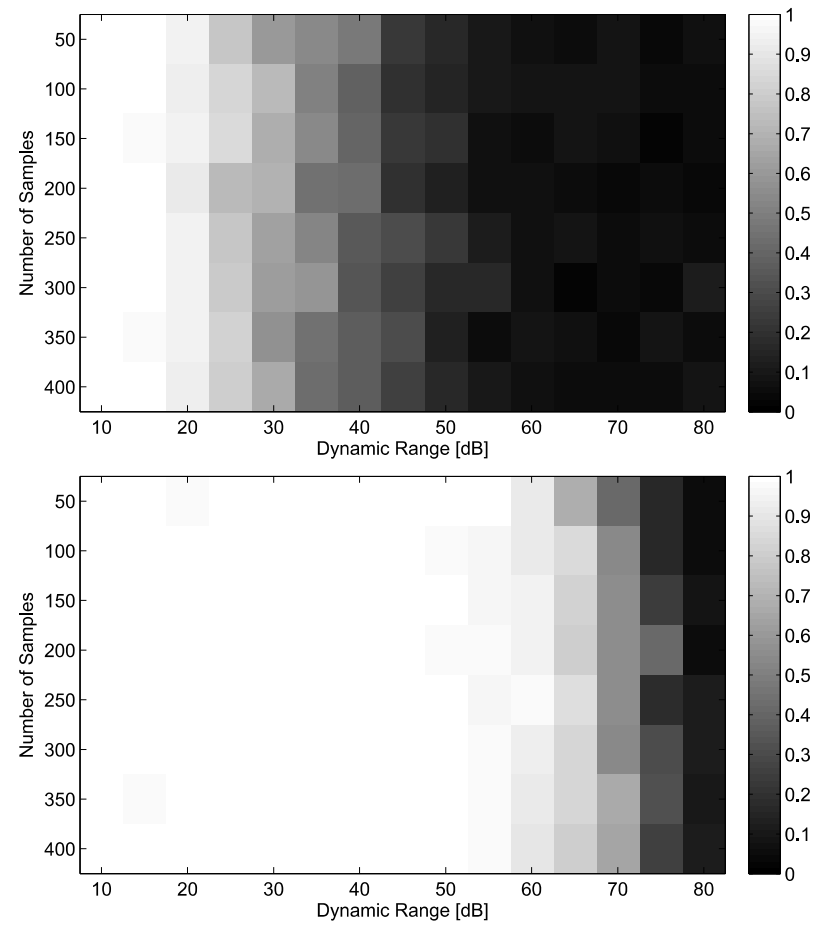

Fig. 4. Probability of correct support recovery as a function of dynamic range and number of samples for without sub-band equalization (top) and with sub-band equalization (bottom).

\section{CONCLUSION}

In this work, we propose an iterative method for equalizing the frequency responses of sub-band channels of the MWC. In each iteration of the proposed method, FIR filters for equalizing the total frequency of each sub-band are designed jointly. The filter design procedure is constructed as a simple least-squares problem. The method converges to fixed filter coefficients in a few iterations hence the method is fast. Obtained results on computer simulations show that the proposed method successfully equalizes the sub-band frequency responses of the MWC and improves its dynamic range significantly and makes MWC useful in real hardware implementations.

\section{REFERENCES}

[1] M. Mishali and Y. Eldar, From Theory to Practice: Sub-Nyquist Sampling of Sparse Wideband Analog Signals, IEEE Journal of Selected Topics in Signal Processing, vol. 4, no. 2, 2010.

[2] M. Mishali, Y. Eldar, O. Dounaevsky, E. Shoshan Xampling: Analog to digital at sub-Nyquist rates, IET Circuits, Devises \& Systems, vol. 5, no. $1,2011$.

[3] S. Cotter, B. Rao, K. Engan, K. Delgado, Sparse Solutions to Linear Inverse Problems With Multiple Measurement Vectors, IEEE Transactions on Signal Processing, vol. 53, no. 7, 2005.

[4] J. Tropp, A. Gilbert, Simultaneous Greedy Approximations via Greedy Pursuit, ICASSP 2005.

[5] J. Tropp, A. Gilbert, Blind Multiband Signal reconstruction: Compressed Sensing For Analog Signals, IEEE Transactions on Signal Processing, vol. 57, no. 3, 2009. 